\title{
Fucoxanthin enhances 5-FU chemotherapeutic efficacy in colorectal cancer cells by affecting MMP-9 invasive proteins
}

\author{
Suwisit Manmuan ${ }^{1 *}$, Ponwit Manmuan ${ }^{2}$ \\ ${ }^{1}$ Division of Pharmacology, Faculty of Pharmaceutical Sciences, Burapha University, Chonburi 20131, Thailand. \\ ${ }^{2}$ Department of Intellectual Property, Ministry of Commerce, Nonthaburi 11000, Thailand.
}

\begin{tabular}{l}
\hline ARTICLE INFO \\
\hline Received on: 08/05/2019 \\
Accepted on: 09/09/2019 \\
Available online: 03/12/2019
\end{tabular}

Key words:

Fucoxanthin, cancer invasion, metastasis, cell adhesion, colorectal cancer.

\begin{abstract}
Drug resistance is a major inconvenience which lowers the traditional chemotherapeutic efficacy and is a highly undesirable therapeutic problem which poses particular challenges in the case of colorectal cancer. Fucoxanthin is a natural orange-carotenoid, predominantly found in edible brown algae and justifiably considered as a nutritional ingredient with the capacity to powerfully enhance concurrent drug chemotherapy. It has been well-documented that fucoxanthin has good potential for anti-cancer activity while offering a remarkable range of biological activities. Accordingly, it has gained prominence in the research field as interest grows in the molecular mechanism which is associated with cancer therapy. This study was undertaken to assess the anti-cancer activity and to explore the molecular mechanism of fucoxanthin on the inhibition of cell proliferation, cell adhesion, and cell invasion, in addition to determining the synergistic effect of drug-drug combinative treatment in colorectal cancer cells. SW-620 cells were cultivated with fucoxanthin for 24, 48, 72, and 96 hours with co-treatment by 5-FU to evaluate the synergistic potential. The cell viability of cancerous cells was determined by MTT colorimetric assay. The inhibitory effects of cell invasion and adhesion were measured in the presence of fucoxanthin with 5-FU in various concentrations to determine MMP-9 gene and protein expression after treatment of the cells by RT-PCR and ELISA assay. The results illustrated that fucoxanthin profoundly inhibited cell proliferation of SW-620 cells, accompanied by arrested growth and diminished invasive ability, which was mediated at least in part by the down-regulation of MMP-9, mRNA, and protein expression. In particular, fucoxanthin strongly attenuated the anti-proliferative effect of established 5-FU by modulating the habitual hallmark of cancerous cells. These results illustrate the capacity of fucoxanthin to eradicate cancer cells and indicate the possibility that fucoxanthin could serve as a promising natural marine product derived from seaweed. The critical data in our studies will serve as the preliminary results for further studies of marine drugs in both experimental models and well-controlled clinical trials.
\end{abstract}

\section{INTRODUCTION}

Colorectal cancer (CRC) is a cancer type that is aberrantly generated in the Thai population and is closely linked to the rapidly increasing incidence rates of morbidity in malignant neoplasia (Sy et al., 2018). As described in the National Comprehensive Cancer Network Guidelines (2018), patients are guided towards regimens which are commonly used as chemotherapy regimens for

\section{"Corresponding Author}

Suwisit Manmuan, Division of Pharmacology, Faculty of Pharmaceutical Sciences, Burapha University, Chonburi 20131, Thailand.

E-mail: suwisit@ buu.ac.th
CRC including 5-FU/LV, Capecitabine, CAPEOX, FOLFIRI, and FOLFOX (National Comprehensive Cancer Network Guidelines for colorectal cancer, 2018). Generally, 5-fluorouracil (5-FU), an analog of uracil, is still the most frequently used chemotherapy drug, which is slowly injected into a vein and selected as the first-line chemo-sensitizing drug to eradicate the characteristics developing in cancerous tissues (Moon et al., 2007). Antimetabolites of 5-FU work preferentially as inhibitors of essential biosynthetic processes through the inhibition of thymidylate synthase and is incorporated into DNA and RNA after rapidly facilitating transportation into cancer cells (Parker, 1990). It is one of a minority of mainstay chemotherapeutic drug which is toxic across a broad spectrum of normal and cancer cells (Latchman, 2014). The well-established 5-FU toxicity may be exacerbated by 
drugs that diminish the major metabolizing enzyme responsible for 5-FU metabolism (Zhang et al., 2017). Modulation strategies, such as co-treatment with oxaliplatin and leucovorin, have been used as co-chemotherapy but are insufficient due to drug resistance in clinical applications (Shin et al., 2005). Therefore, the screening of marine natural products capable of increasing the functional potential of 5-FU used as a chemotherapeutic drug or possibly serving as a cell cycle arrest agent similar to 5-FU is now needed. The anti-cancer activities of drugs and natural compounds involve the induction apoptosis pathway which is accompanied by cell cycle arrest (Murad et al., 2016).

Numerous effective marine anti-cancer drugs have been discovered from natural sources (Lindequist, 2016). Fortunately, marine natural products from algae as nutraceutical substances are currently available in the marine environment (Zhang et al., 2015). Characterization of the active ingredients of marine products can be evaluated in the literature review, which describes the abundant bioactive activities and the fact that such substances have received increasing attention from scientific researchers. Fucoxanthin is an orange-colored pigment which is ordinarily isolated from brown seaweeds and is understood to be a new destiny carotenoid with potential applications in human health (Rengarajan, 2013). It is a special carotenoid which can be easily purified from macroalgae and microalgae. Fucoxanthinol is the main digestive metabolite of fucoxanthin after oral administration (Maeda, 2015). The chemical structures of fucoxanthin and fucoxanthinol are represented in Figure 1. Fucoxanthin has certain biological properties, including anti-cancer, anti-inflammation, anti-diabetic, and anti-angiogenic activities (Martin, 2015).

Many journals have represented the molecular mechanism of fucoxanthin, which is mediated through the interference of the cell cycle progression and acts as an apoptosis inducer in the generation of apoptosis in various types of cancer. In recent journals, it was reported that fucoxanthin caused remarkable reductions in the general viability of human colon cancer cell lines, such as Caco-2, HT-29, and DLD-1. Therefore, treatment with fucoxanthin can induce DNA fragmentation leading to apoptosis. Moreover, combined treatment with low doses of fucoxanthin and troglitazone, which is a specific ligand for the peroxisome proliferator-activated receptor (PPAR), strongly repressed the

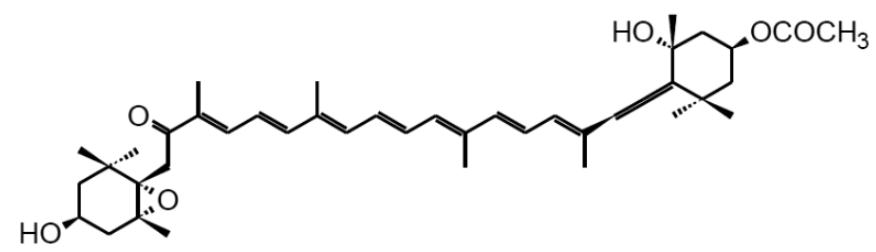

(a)

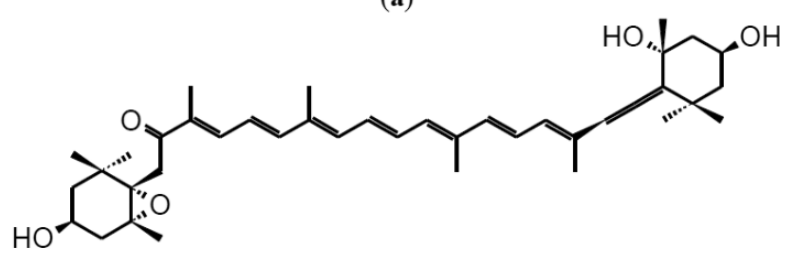

(b)

Figure 1. Chemical structures of (a) Fucoxanthin and (b) Fucoxanthinol. viability of Caco- 2 cells. These data indicated that fucoxanthin may act as a chemo-preventive and chemotherapeutic regimen which is responsible for attacking colon cancer cells by changing cell viability in combination with troglitazone (Hosokawa, 2004). In previous studies demonstrated that fucoxanthin induced cell cycle arrest at the $G_{0} / G_{1}$ phase of the cell cycle via the upregulation of $\mathrm{p} 21 \mathrm{WAF} 1 / \mathrm{Cip} 1$ in human colon carcinoma cells. The result demonstrated that fucoxanthin decreased the viability of WiDr cells accompanied by the induction of apoptosis and modulation of the cell cycle progression by arresting cells in the $\mathrm{G}_{0} / \mathrm{G}_{1}$ phase, suppressing the phosphorylation of the retinoblastoma protein $(\mathrm{pRb})$ at Ser780 and Ser807/811, for which the underlying mechanism may be sharply related to the anti-tumorigenic activity. This phenomenon is a critical event for cells in maintaining their viability, leading to cellular disassembly and serving as a marker of cells undergoing apoptosis (Das et al., 2005). Consistently, fucoxanthin induced apoptosis by ROS generation and mediated one possible pathway through the activation of the Bcl-xL pathway in human leukemia HL-60 cells. The results revealed that fucoxanthin actually generated the accumulation of reactive oxygen species (ROS) and triggered the Bcl-xL signaling pathway which significantly activated HL-60 cells apoptosis. Moreover, fucoxanthin has been shown to play a crucial role in activating the cleavage of caspases -3 and -7 , and poly-ADP-ribose polymerase (Kim, 2010). Additionally, fucoxanthin induced HeLa cervical cancer cell apoptosis by the inhibition of the PI3K/Akt pathway. Moreover, fucoxanthin represses the mitochondrial signal transduction pathway and NF- $\kappa \mathrm{B}$ activation, which is associated with the development of cervical cancer (Ye, 2014). The present study is focused on the anti-cancer activity of fucoxanthin and its effects on cell growth, and the invasive activity of fucoxanthin on SW-620 colorectal cancer cells.

In summary, fucoxanthin demonstrates the good potential to enhance the 5-FU chemotherapeutic efficacy in colorectal cancer cells. When fucoxanthin is combined with 5-FU, additive killing is demonstrated and its mechanism of action on cell invasion at the gene and protein levels is determined. These findings suggest a new strategy to combine fucoxanthin with 5-FU as a drug combinative regimen, to enhance the therapeutic ratio and increase the drug response rate as well as generating highly therapeutic outcomes in colorectal cancer.

\section{MATERIALS AND METHODS}

\section{Natural compounds and drug synthesizing agents}

Fucoxanthin was purchased from the Sigma Chemical Company (St. Louis, MO). Fucoxanthin was prepared from the stock solution at the concentration of $10 \mathrm{M}$ by dissolving in absolute DMSO and storing at $-20^{\circ} \mathrm{C}$. 5 -FU was purchased from Sigma-Aldrich and dissolved in DMSO to adjust the concentration of the stock solution to $10 \mathrm{M}$.

\section{Chemicals and reagents}

The chemicals and reagents used in this study included $0.25 \%$ trypsin-Ethylene diamine tetra-acetic acid (EDTA) (Gibco, USA), 0.4\% trypan blue (Sigma, USA), Dimethyl sulfoxide (DMSO) (Sigma, USA), RPMI-1640 medium (Gibco, USA), Fetal bovine serum (Gibco, USA), Penicillin-streptomycin (Sigma, 
USA), 96-well plate (Corning, USA), 24-well plate (Corning, USA), Matrigel invasion chambers (Corning, USA), BD matrigel matrix (Biosciences, USA), T-25 cell culture flasks (Corning, USA), Fungizone $^{\circledR}$ (Gibco, USA), and 3-(4,5-dimethylthiazol-2-yl)-2,5diphenyltetrazolium bromide (MTT reagent) (Sigma, USA).

\section{Cell cultures}

SW-620 colorectal cancer cells were obtained from the American Type Culture Collection (ATCC ${ }^{\circledR}$ Number: CCL-227 ${ }^{\mathrm{TM}}$ ). The SW-620 cells were routinely propagated in RPMI-1640 medium and supplemented with $10 \%$ heat-inactivated fetal bovine serum (FBS), $1 \%$ penicillin and streptomycin, and amphotericin B at $37^{\circ} \mathrm{C}$ in a $5 \%$ atmosphere of $\mathrm{CO}_{2}$ in the $\mathrm{T}-25$ cell culture flask. When the cell confluences reached around $90 \%$, the cells were fully trypsinized by trypsin-EDTA to harvest the adhering cells. Then, $200 \mu \mathrm{l}$ of cancer cells were added into the culture flask to allow attachment. The cells were routinely sub-cultured every 3 days to maintain the genetic stability.

\section{Cell viability assay}

Based on the reduction of yellow tetrazolium solution to purple formazan crystals using mitochondrial dehydrogenase enzymes in viable cells, the MTT viability assay was typically used as a precise method to determine the viable cancerous cells. SW-620 cells at a density of 5,000 cells/well were trypsinized by trypsin-EDTA to harvest the adhered cells in the cell culture flask. Cells for which the number was actually known for cell seeding were specifically pipetted into the 96-well plate. Cells were then treated with $10,15,20,25$, and $30 \mu \mathrm{M}$ of fucoxanthin for 24, 48, 72, and 96 hours. After the incubation period, MTT in phosphate-buffered saline (PBS) solution was added into each well to determine the yield of the total reaction in viable cells by incubating the plate for 3 hours. The supernatants were aspirated and $100 \mu \mathrm{l}$ of absolute DMSO was added to dissolve the formazan crystals. The plates were read with a scanning microplate reader (Metertech, Taiwan) at $550 \mathrm{~nm}$ to receive the data after exposure to fucoxanthin.

\section{Drug combination assay}

Drug combinative experiments were carried out by MTT assay with a slight modification. Cells were pipetted at a density of 5,000 cells/well into a 96 -well plate and placed at $37^{\circ} \mathrm{C}$ in a $5 \%$ $\mathrm{CO}_{2}$ atmosphere for 24 hours. The medium was then aspirated from each well. The cells were co-treated with $10 \mu \mathrm{M}$ fucoxanthin and $10 \mu \mathrm{M}$ fucoxanthin in the presence of 5-FU at the concentrations of $0.1,0.5,1.5$, and $2.5 \mu \mathrm{M}$ of 5 -FU for 36 hours. Following the drug exposure period, $10 \mu \mathrm{l}$ of MTT in PBS solution was added to develop formazan crystals which were solubilized by absolute DMSO. The plates were analyzed using a scanning microplate reader (Metertech, Taiwan) at a wavelength of $550 \mathrm{~nm}$. The purple density of the crystals after solubilization with DMSO is directly proportional to the number of living cells.

\section{Cell adhesion assay}

Cells at a density of 5,000 cells/well were pre-incubated with $10 \mu \mathrm{M}$ fucoxanthin and $10 \mu \mathrm{M}$ fucoxanthin in the presence of 5-FU at the concentrations of $0.1,0.5,1.5$, and $2.5 \mu \mathrm{M}$ of $5-\mathrm{FU}$ for 24 hours. Cells were seeded into a 96-well plate coated with matrigel for 4 hours at $37^{\circ} \mathrm{C}$. Non-attached cells were removed by PBS. Attached cells were then suddenly fixed with cold absolute methanol (AMRESCO, USA) and stained with crystal violet (Sigma, USA) for 5 minutes at room temperature. Randomly chosen fields were captured under an inverted microscope (Olympus, Japan). The number of adhesive cells was manually counted and expressed as a percentage of cell adhesion.

\section{Transwell invasion assay coated with matrigel}

SW-620 cells at a density of 500 cells were harvested and resuspended in RPMI-1640 medium with 1\% FBS for 2 minutes. The lower chambers of the transwell were fully filled with $500 \mu \mathrm{l}$ of RPMI-1640 medium to act as chemo-attractants in the system. The cells were seeded in the upper chamber of the experimental system in the presence of $10 \mu \mathrm{M}$ fucoxanthin and $10 \mu \mathrm{M}$ fucoxanthin in the presence of 5-FU at the concentrations of $0.1,0.5,1.5$, and 2.5 $\mu \mathrm{M}$ of 5-FU for 48 hours. The upper chambers were wiped off and swabbed with a cotton swab to remove the uncounted cells. Cells which crossed through the matrigel-coated invasion chamber were fixed with ice-cold methanol to quantify the number of cells which migrated. Crystal violet was used for staining to measure the attached cells in the invasion chamber. The cell attachment in the invasion chamber was randomly photographed with an inverted microscope (Olympus, Japan). Data were presented as a percentage of cell invasion through the matrigel-coated invasion chamber.

\section{Reverse transcriptase polymerase chain reaction (RT-PCR)}

SW-620 cells were added into the RPMI-1640 medium at a density of $1 \times 10^{6}$ cells $/ \mathrm{ml}$ in each well of a 24 -well plate and incubated at $37^{\circ} \mathrm{C}$ in an incubator with $5 \% \mathrm{CO}_{2}$ for 24 hours. The cells were treated with $10 \mu \mathrm{M}$ fucoxanthin and $10 \mu \mathrm{M}$ fucoxanthin in the presence of 5-FU at the concentrations of $0.1,0.5,1.5$, and $2.5 \mu \mathrm{M}$ of 5 -FU for 48 hours in the $\mathrm{CO}_{2}$ incubator. The total RNA was wholly extracted using PureLink ${ }^{\mathrm{TM}}$ RNA Mini Kit. The total RNA was then transcribed to cDNA by the ImProm-11 ${ }^{\mathrm{TM}}$ reverse transcription system. The cDNA was used as the template to amplify mRNA with specific primers using PCR thermal FlexCycler ${ }^{2}$ (Life Science, Germany). The PCR cycle began with an initial denaturation process at $95^{\circ} \mathrm{C}$ for 1 minute, followed by 30 cycles of denaturation at $95^{\circ} \mathrm{C}$ for 1 minute, annealing at $55^{\circ} \mathrm{C}$ for 1 minute, with extension at $72^{\circ} \mathrm{C}$ for 1 minute, and final extension at $72^{\circ} \mathrm{C}$ for 5 minutes. The PCR products were run on $1.5 \%$ agarose gel electrophoresis. Total gel was stained with ethidium bromide to determine the densities by gel documentation (GelMax imager, UVP, UK) and Image Lab ${ }^{\mathrm{TM}}$ software. Primer sequences used in RT-PCR were presented as follows: MMP-9: 5'-TCG-AAG-GCG-ACC-TCA-AGT-G-3' and 5'-TTC-GGT-GTA-GCT-TTG-GAT-CCA-3'; GAPDH: 5'-AGGGCT-GCT-TTT-AAC-TCT-GGT-3' and 5'-CCC-CAC-TTG-ATTTTG-GAG-GGA-3'. Primer sequencing was performed by the Macrogen DNA Sequencing Service, Thailand.

\section{MMP-9 immunosorbent assay (ELISA)}

MMP-9 protein contents were determined following manufacturing protocol (Invitrogen, U.S.A.). SW-620 cells were carefully resuspended at a density of 50,000 cells/well in a 24-well plate for 24 hours. Cells were cultured with $10 \mu \mathrm{M}$ fucoxanthin and $10 \mu \mathrm{M}$ fucoxanthin in the presence of 5-FU at the concentrations 
of $0.1,0.5,1.5$, and $2.5 \mu \mathrm{M}$ of 5 -FU for 48 hours. The supernatants were collected from the culture plates. Supernatants were then diluted with standard diluent buffer (1:20). The sample volume was added into each well and covered with aluminum foil. The plates were then incubated at room temperature for 2 hours before the addition of $100 \mu \mathrm{l}$ of biotinylated Hu MMP-9 Biotin conjugate solution, streptavidin-HRP working solution, and stabilized chromogen. Finally, $100 \mu \mathrm{l}$ of stop solution was added to stop the reaction which appeared in each well, observable by the blue color changing to a yellow color. The MMP-9 protein in the serums was directly evaluated by the microplate reader at $450 \mathrm{~nm}$. MMP9 concentrations were determined by comparison with graph standard curves. The MMP-9 protein levels were shown as $\mathrm{pg} / \mathrm{ml}$.

\section{Statistical analysis}

The data were obtained from at least three experiments $(n=3)$ and the data were presented in the form of mean \pm SEM and determined by one-way analysis of variance with Tukey's
Honestly Significant Difference post-hoc test. The $p$-values less than 0.05 were considered as statistically significant compared to the control group (untreated control). Graphs were plotted using GraphPad Prism software Ver.7.0. (GraphPad Software, San Diego, CA).

\section{RESULTS}

\section{Inhibitory effect of fucoxanthin on cell viability of colorectal cancer cells}

The cytotoxicity of fucoxanthin against the cancer cells was investigated via MTT assay. Fucoxanthin demonstrates a significant inhibitory effect on the proliferation of SW-620 cells in doses- and time-dependent manners, with inhibition rates of $22.2 \%, 32.5 \%, 43.2 \%, 54.1 \%$, and $75.2 \%$ at $10,15,20,25$, and $30 \mu \mathrm{M}$, respectively, after treatment for 24 hours. The low concentrations of fucoxanthin arrested the cell proliferation and the higher concentrations $(20,25$, and $30 \mu \mathrm{M})$ markedly
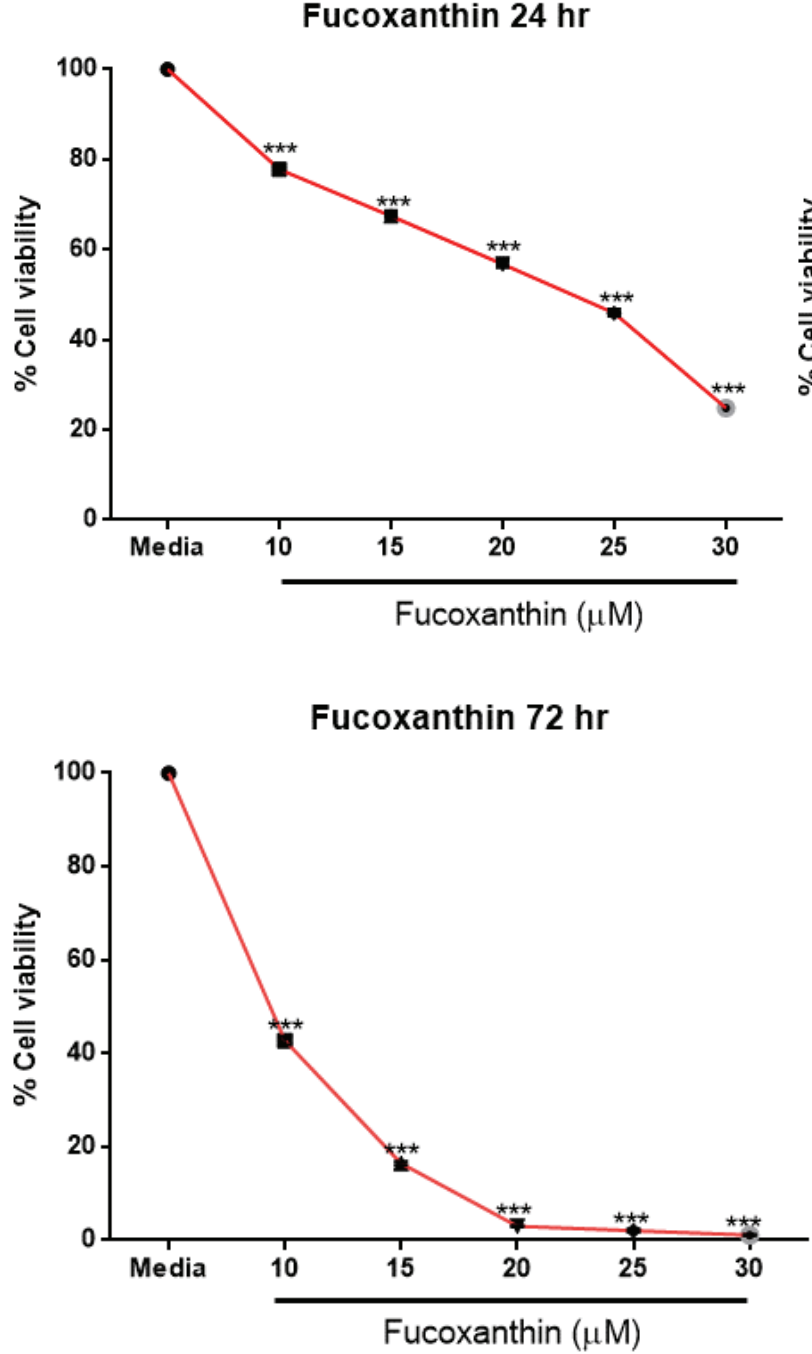

\section{Fucoxanthin $48 \mathrm{hr}$}

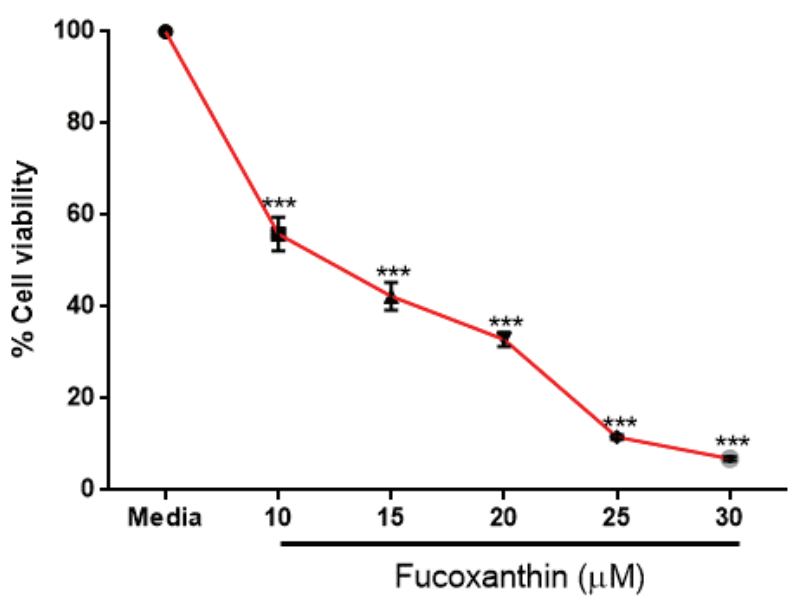

Fucoxanthin $96 \mathrm{hr}$

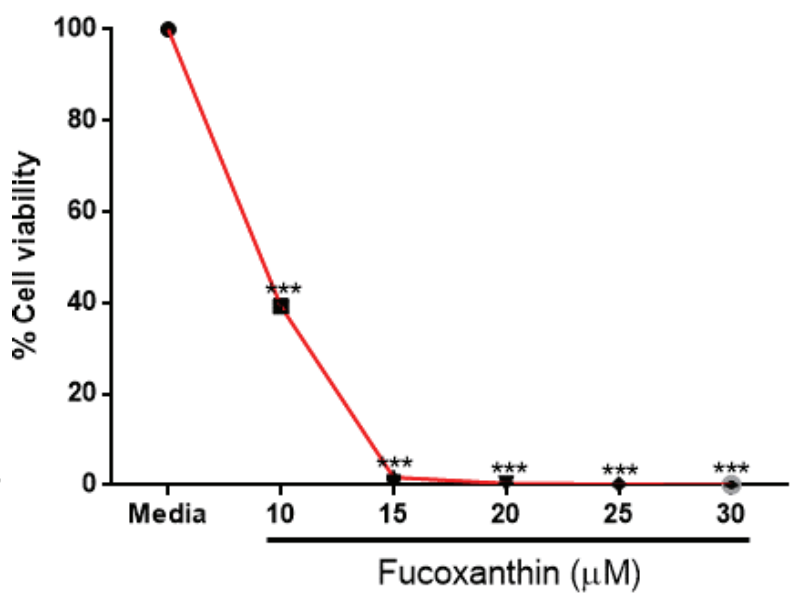

Figure 2. The survival analysis of SW-620 cells treated with fucoxanthin. SW-620 cells were seeded at 5,000 cells/well and treated with various concentrations of fucoxanthin for $24,48,72$, and 96 hours. Cell survival was measured with an MTT cell viability assay. The data were represented as mean \pm SEM from at least three independent experiments. ${ }^{* * *}$ Significant difference from control $(p<0.001)$. 
decreased the ability of cancer cells after treatment for 48, 72, and 96 hours. The $\mathrm{IC}_{50}$ values after cells were exposed to fucoxanthin for 24, 48, 72, and 96 hours are $21.74 \pm 0.0488,13.95 \pm 0.510$, $9.911 \pm 0.096$, and $8.674 \pm 0.111 \mu \mathrm{M}$, respectively. Therefore, the dietary fucoxanthin may be used to specifically suppress cancer cells.

To calculate the inhibitory concentration of fucoxanthin on cell viability of SW-620 colorectal cancer cells after treatment for 24, 48, 72, and 96 hours

\begin{tabular}{|c|c|c|c|}
\hline \multirow{2}{*}{$\begin{array}{c}\text { Cell lines (SW-620) } \\
\text { Times }\end{array}$} & \multicolumn{3}{|c|}{$\begin{array}{l}\text { Inhibitory concentration of fucoxanthin at } 25 \%, 50 \% \text {, } \\
75 \%\end{array}$} \\
\hline & $\mathrm{IC}_{25}(\mu \mathrm{M})$ & $\mathrm{IC}_{50}(\mu \mathrm{M})$ & $\mathrm{IC}_{75}(\mu \mathrm{M})$ \\
\hline 24 & $11.29 \pm 0.063$ & $21.74 \pm 0.0488$ & $32.19 \pm 0.035$ \\
\hline 48 & $5.891 \pm 0.579$ & $13.95 \pm 0.510$ & $22.00 \pm 0.445$ \\
\hline 72 & $2.380 \pm 0.111$ & $9.911 \pm 0.096$ & $17.44 \pm 0.081$ \\
\hline 96 & $1.106 \pm 0.149$ & $8.674 \pm 0.111$ & $16.24 \pm 0.072$ \\
\hline
\end{tabular}

***Each value was represented as mean \pm SEM from three independent experiments $(n=3)$.

Fucoxanthin attenuates the efficacy of 5-FU and increases the sensitivity to 5-FU in colorectal cancer cells

The synergistic drug combination assay demonstrated that the cell viability of SW-620 colorectal cancer cells was significantly decreased after co-treatment with a low dose of 5-FU
$(0.5,1.5$, and $2.5 \mu \mathrm{M})$ and varying doses of fucoxanthin (10, $15,20,25$, and $30 \mu \mathrm{M})$. These results indicated that fucoxanthin concurrently attenuated the potential of 5-FU as the exhibited drug-interaction confirms the synergistic effect. Therefore, the combinative regimens are of great interest for further study to examine syncytial efficacy in eliminating metastatic colorectal cancer.

\begin{tabular}{|c|c|c|c|}
\hline \multirow{2}{*}{$\begin{array}{l}\text { Cell lines (SW-620) } \\
\text { Times } 36 \text { hours }\end{array}$} & \multicolumn{3}{|c|}{$\begin{array}{l}\text { Inhibitory Concentration of Fucoxanthin }+5 \text {-FU at } \\
\qquad 25 \%, 50 \%, 75 \% \%\end{array}$} \\
\hline & $\operatorname{IC25}(\mu \mathrm{M})$ & IC50 $(\mu \mathrm{M})$ & IC75 $(\mu \mathrm{M})$ \\
\hline Fucoxanthin $+2.5 \mu \mathrm{M} 5$-FU & $2.288 \pm 0.124$ & $9.735 \pm 0.101$ & $17.18 \pm 0.082$ \\
\hline Fucoxanthin $+1.5 \mu \mathrm{M} 5$-FU & $6.057 \pm 0.138$ & $13.27 \pm 0.115$ & $20.49 \pm 0.093$ \\
\hline Fucoxanthin $+0.5 \mu \mathrm{M} 5$-FU & $6.741 \pm 0.355$ & $14.21 \pm 0.540$ & $21.68 \pm 0.745$ \\
\hline
\end{tabular}

***Each value was represented as mean \pm SEM from three independent experiments $(n=3)$.

Fucoxanthin combined with 5-FU represses adhesion ability and invasive power in colorectal cancer cells

The results showed that fucoxanthin at $10 \mu \mathrm{M}$ has an anti-adhesive effect with the percentage of cell adhesion reported as $77.61 \%$. Moreover, fucoxanthin at $10 \mu \mathrm{M}$ plus 0.1 , $0.5,1.5$, and $2.5 \mu \mathrm{M}$ of $5-\mathrm{FU}$ has an additive synergistic effect
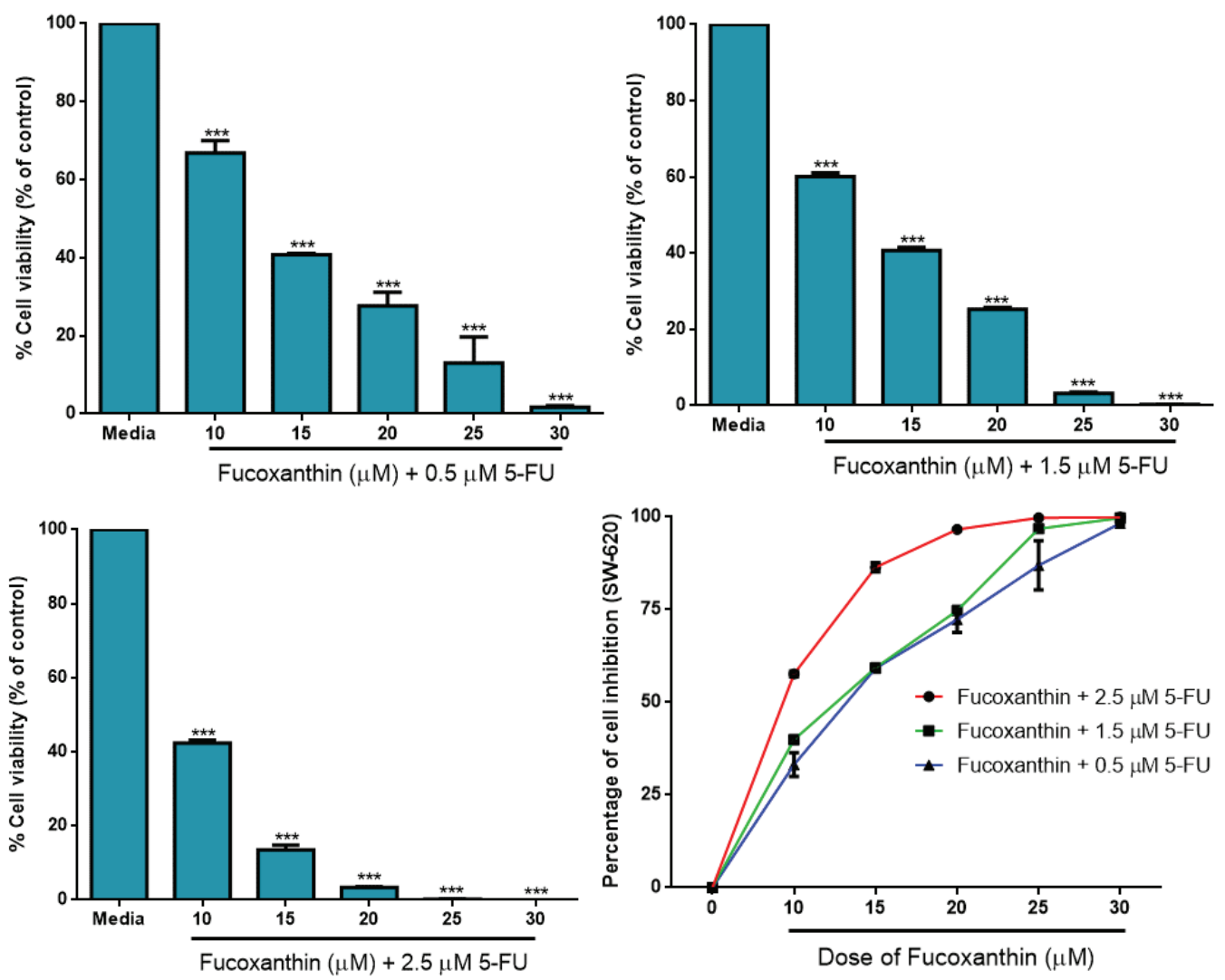

Figure 3. Fucoxanthin attenuates the anti-proliferative activity of 5-FU on colorectal cancer cells. Fucoxanthin was added to SW620 cells at concentrations of $10,15,20,25$, and $30 \mu \mathrm{M}$ in the presence of 5-FU for 36 hours. Cell viability in the treated wells was directly determined using MTT assay. ***Significant difference from control $(p<0.001)$. 

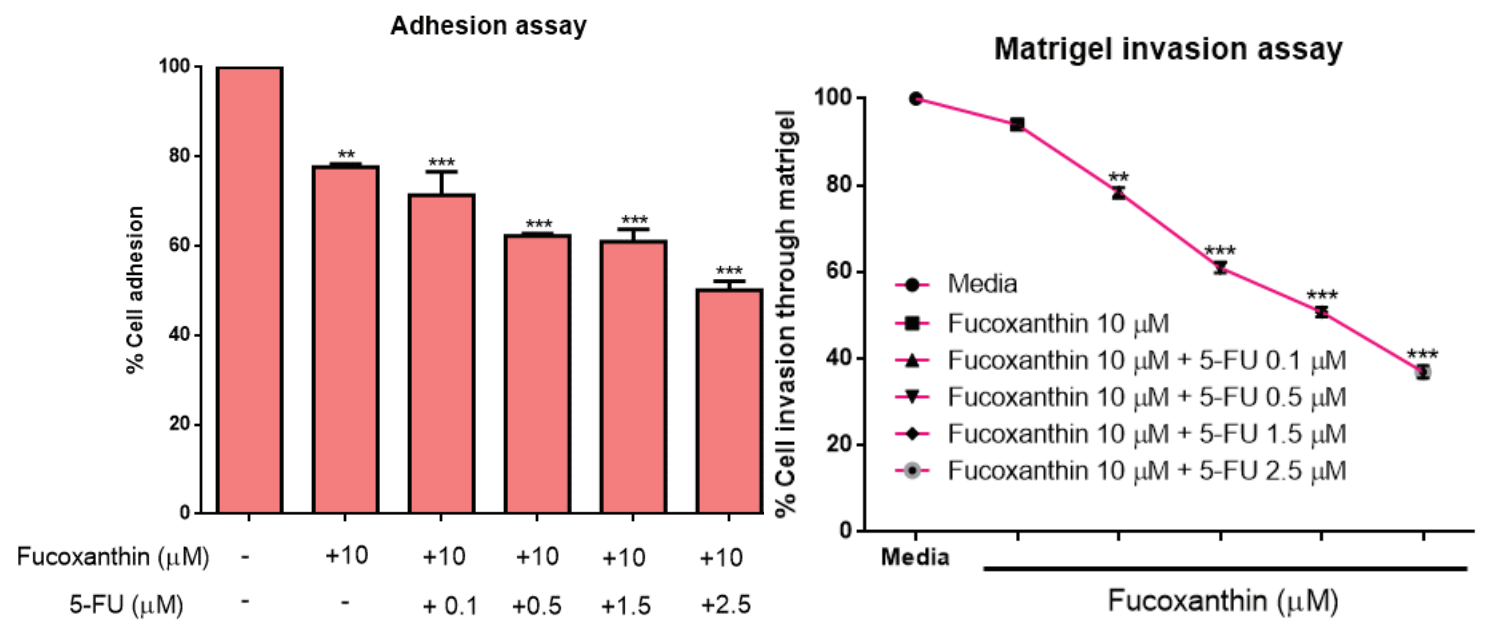

Figure 4. The effect of fucoxanthin alone and in combination with 5-FU on cell adhesion and cell invasion in SW-620 colorectal cancer cells. SW-620 cells were incubated with $10 \mu \mathrm{M}$ fucoxanthin and $10 \mu \mathrm{M}$ fucoxanthin in the presence of 5-FU at the concentrations of $0.1,0.5,1.5$, and $2.5 \mu \mathrm{M}$ of 5-FU for 24 hours and 48 hours. Adhesive and invasive cells were randomly captured and manually counted in five random fields using a microscope. The experiments were performed in triplicate $(n=3) * *$ Significant differences at $p<0.01$, $* * *$ Significant differences at $p<0.001$.

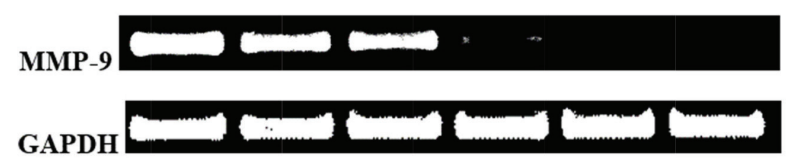

Fucoxanthin $(\mu \mathrm{M}) \quad-\quad+10 \quad+10 \quad+10 \quad+10 \quad+10$ $5-\mathrm{FU}(\mu \mathrm{M}) \quad-\quad-\quad+0.1+0.5+1.5+2.5$

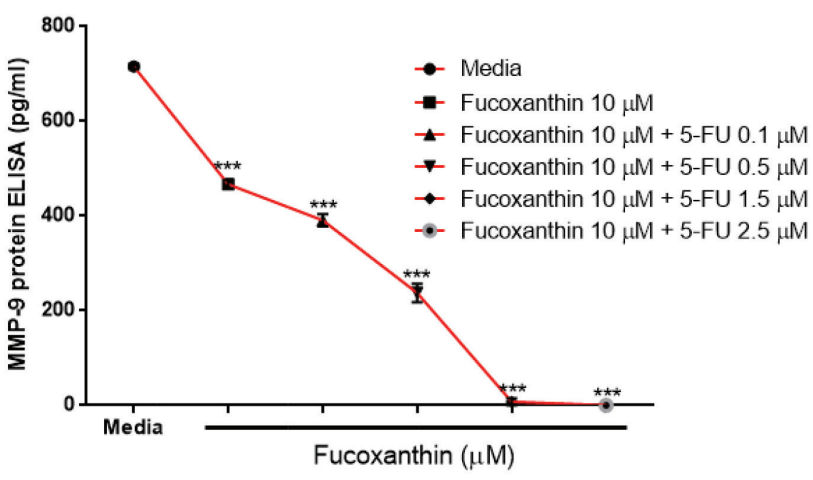

\begin{tabular}{lcccccc}
\hline \multirow{2}{*}{$\begin{array}{l}\text { Serum } \\
\text { MMP-9 } \\
\text { protein }\end{array}$} & Media & \multicolumn{5}{c}{ Fucoxanthin $\mathbf{1 0} \boldsymbol{\mu M}$} \\
\cline { 3 - 7 } & & - & $+\mathbf{5 - F U}$ & $+\mathbf{5 - F U}$ & $+\mathbf{5 - F U}$ & $+\mathbf{5 - F U}$ \\
& & & $\mathbf{0 . 1} \boldsymbol{\mu M}$ & $\mathbf{0 . 5} \boldsymbol{\mu} \mathbf{M}$ & $\mathbf{1 . 5} \boldsymbol{\mu M}$ & $\mathbf{2 . 5} \boldsymbol{\mu M}$ \\
\hline Mean & 715.8 & 466.9 & 391.2 & 237.5 & 7.50 & 0.0 \\
SEM & 6.155 & 8.850 & 13.080 & 19.770 & 7.500 & 0.000 \\
\hline
\end{tabular}

Figure 5. The effect of fucoxanthin alone and in combination with 5-FU on mRNA and protein expression of MMP-9 in SW-620 colorectal cancer cells. SW-620 cells were treated with $10 \mu \mathrm{M}$ fucoxanthin and $10 \mu \mathrm{M}$ fucoxanthin in the presence of 5-FU at the concentrations of $0.1,0.5,1.5$, and $2.5 \mu \mathrm{M}$ for 48 hours. The total RNA was extracted from the treated cells and reverse transcribed for amplification with specific primer by RT-PCR. The PCR products were run on $1.5 \%$ agarose gel and analyzed by gel documentation. The protein content in culture serum was determined by ELISA. The experiments were performed in triplicate $(n=3)$. ${ }^{* * *}$ Significant differences at $p<0.001$. on cell adhesion with the percentages of cell adhesion reported, respectively, as $71.37 \%, 62.24 \%, 60.94 \%$, and $50.12 \%$ when compared to the untreated cells. To explore the antinvasive effect of fucoxanthin on cancer cells, assessment was carried out by matrigel invasion assay. The results demonstrated that $10 \mu \mathrm{M}$ fucoxanthin in combination with $0.1,0.5,1.5$, and $2.5 \mu \mathrm{M}$ of 5-FU additively inhibited the percentage of invasiveness of SW-620 cells after treatment for 72 hours. The percentages of cell invasion through matrigel were $93.99 \%, 78.40 \%, 61.01 \%$, $50.76 \%$, and $36.95 \%$, respectively. Therefore, fucoxanthin may be used as an anti-invasive agent when used in treatments with 5-FU in colorectal cancer patients.

\section{Abrogation of MMP-9 mRNA and protein expression by fucoxanthin and 5-FU in colorectal cancer cells}

Based on the role and function of MMP-9 in pathological conditions, the development of effective therapy by selective targeting of MMP-9 is the ultimate goal of colorectal cancer treatments. RT-PCR results demonstrated that fucoxanthin sufficiently down-regulated MMP-9 mRNA expression after coincubating with 5-FU in SW-620 cells. The results from the MMP9 ELISA assay demonstrated that co-treatment with fucoxanthin plus 5-FU was able to sharply decrease MMP-9 protein levels from 466.91 to $0 \mathrm{pg} / \mathrm{ml}$. These regimens may constitute the valuable active ingredients in the combination therapy of highly adhesive colorectal cancer cells.

\section{DISCUSSION AND CONCLUSION}

Cytotoxic chemotherapy has long been recognized and prescribed by clinicians for the treatment of CRC patients. Fluoropyrimidines (FPs) or 5-FU have been extensively used as backbone chemotherapy and incorporated into practical guidelines as combinative drug regimens, leading to widespread use in clinical settings for drug co-chemotherapy (Mukai et al., 2009). However, 
the adoption of 5-FU eventually occurred in the advanced stage (III, IV) of colorectal cancer and it could be identified that some patients are intrinsically resistant to 5-FU, which is characterized by cancer cells having only a slight response or unsustainable active response to treatment. This is due to the development of resistance which has been pre-manifested in patients (Hammond, 2016). Different mechanisms, which eventually contribute to traditional cytotoxic therapeutic resistance, are typically accomplished by generating drug efflux pumps to diminish the delivery of the drug to the target site inside the cancer cell, or by changing the enzymes involved in drug metabolism. Alternately, multi-drug resistance can be generated by genetic and epigenetic modification leading to altered drug sensitivity and mediating at one least mechanism by ATP binding cassette $(\mathrm{ABC})$ protein transporters, responsible for the decreased drug uptake into the cancer cells and exerting their mode of action through diminishing the intracellular concentration of the chemotherapeutic drug, which subsequently leads to the generation of drug resistance (Van der Jeught, 2018). The role of $\mathrm{ABC}$ transporters has been postulated in drug resistance and might be instigated via the drug efflux mechanism and can interfere with 5-FU activity to rapidly impede both DNA and RNA synthesis processes. $\mathrm{P}$-glycoprotein (P-gp) is a drug efflux pump, principally active in interfering with the number of targeting drugs and exerting its effects by decreasing the intracellular concentration of anti-cancer drugs, which are subsequently less effective as chemotherapeutic drugs and support drug resistance. Moreover, inactivation of the apoptotic pathway, p53 mutation, and DNA damage repair are the principal rationale in drug resistance. Other resistance mechanisms are preferentially involved in acquired and intrinsic resistance to colorectal cancer treatment, including the Warburg effect, clonal evolution, intra-tumor heterogeneity, and pharmacoepigenomics, which serve as essential novelties in the resistance mechanism (Abraham, 2006; Briffa, 2017). Greater understanding of the molecular mechanism of the drug resistance which is a determinant of drug sensitivity in cancerous cells will lead to rationally designed treatment combinations. Moreover, the identification of novel substances which can be modulated under standard 5-FU cytotoxicity is a one way to solve the specific issue of drug resistance, leading to the restoration of drug sensitivity in cancer cells while cutting out the mechanisms of 5-FU resistance. Therefore, it is important to dissect the mechanism of 5-FU resistance by targeting the use of drug combination therapy because the findings would be of considerable interest (Clarke et al., 2019). The results demonstrated that fucoxanthin is a potent anti-cancer compound extracted from seaweed, which acts against colorectal cancer cells, accomplishing its role by inhibiting cell proliferation and repressing the constitutive ability in cancer growth. Most importantly, we demonstrated that fucoxanthin has a continuous synergistic effect with 5-FU to inhibit cell viability in sensitive colorectal cancer cells or to increase the efficacy of 5-FU to eradicate SW-620 cells. These data suggested that fucoxanthin may be useful for increasing 5-FU sensitivity in colorectal cancer cells resulting in the outgrowth of resistant cells. It will refer solely to salient drug resistance in most colorectal patients. This is the first finding to illustrate the fucoxanthin-attenuated 5-FU mode of action which exerts a better synergistic effect when compared to fucoxanthin treatment alone. These data may be used to recommend the therapeutic approach to manage colorectal cancer patients and may give more insight into the potential of this compound which should be further examined using in vivo study to provide additional benefits in colorectal cancer therapy.

Matrix metalloproteinases (MMPs) is an important enzyme that degrades the extracellular matrix, while collagen type IV and gelatin were the main substrates for these enzymes. In particular, matrix metalloproteinases-9 (MMP-9) or gelatinase B is constitutively expressed in various cell types, including keratinocytes, macrophages, polymorphonuclear leukocytes, and some malignant tumor cells (Mackay et al., 1992; Van den Steen, 2002). MMP-9 can degrade many extracellular matrix (ECM) proteins through proteolytic cleavage and is closely related to ECM remodeling which supports tumor invasion and metastasis. MMP-9 has been identified as a potential cancer biomarker and novelty biosensor (Huang, 2018; Jonsson, 2018). MMP-9 has high levels of expression correlated with worse outcomes and delayed drug response in a majority of colorectal cancer patients. Previous studies found that the serum of MMP2 and MMP-9 proteins was highly expressed with significant concentrations in CRC patients compared to the normal control (Dragutinovic et al., 2011). Hence, MMP-9 is an interestingly attractive therapeutic target in colorectal cancer. We clearly demonstrated for the first time that fucoxanthin in combination with 5-FU significantly down-regulates mRNA and the protein expression of MMP-9 in SW-620 colorectal cancer cells, leading to the suppression of adhesion and invasion of cancerous cells. The findings in this study demonstrated for the first time that fucoxanthin may be suitable as a natural chemotherapeutic compound for clinical application and treatment of colorectal cancer patients as an adjunctive combination therapy with the long-established 5-FU. The combination of fucoxanthin with 5-FU may improve the rates of response to chemotherapy in these patients. However, it requires further investigation in experimental models and clinical settings in the future to clarify its potential.

In conclusion, our present investigation demonstrated that the combination of fucoxanthin and 5-FU exerted better synergistic effect than could be achieved by individual agent treatment, and this synergism which is based on the antihabitual hallmarks of cancer activity through reduction of cell proliferation consequently abrogated the expression of the MMP-9 invasive molecules which assist tumors during angiogenesis, giving support to the migrative capacity of cancer cells. This study also provided preclinical data showing that the combinative regimen could be a novel and promising therapeutic approach to manage advanced colorectal cancer, which must, therefore, warrant further investigation of its efficacy and advantages, in addition to highlighting its practical applications through a clinical study.

\section{ACKNOWLEDGMENT}

This project was fully supported by a Research Grant from Burapha University through the National Research Council of Thailand (Grant number 18/2559 and Grant number 169/2561) for Suwisit Manmuan. We also thank the Faculty of Pharmaceutical Sciences, Burapha University, for support through the provision of laboratory facilities and general equipment for the research. 


\section{CONFLICT OF INTEREST}

There is no conflict of interest in the present study.

\section{REFERENCES}

Abraham J, Earl HM, Pharoah PD, Caldas C. Pharmacogenetics of cancer chemotherapy. Biochim Biophys Acta, 2006; 1766(2):168-83.

Briffa R, Langdon PS, Grech G, Harrison DJ. Acquired and Intrinsic Resistance to colorectal cancer treatment, colorectal cancer diagnosis, screening and management. IntechOpen, Jindong Chen. doi: 10.5772/intechopen.70781. Available via https://www.intechopen.com/ books/colorectal-cancer-diagnosis-screening-and-management/acquiredand-intrinsic-resistance-to-colorectal-cancer-treatment

Clarke PA, Roe T, Swabey K, Hobbs SM, McAndrew C, Tomlin K, Westwood I, Burke R, van Montfort R, Workman P. Dissecting mechanisms of resistance to targeted drug combination therapy in human colorectal cancer. Oncogene, 2019; 38(25):5076-90.

Das SK, Hashimoto T, Shimizu K, Yoshida T, Sakai T, Sowa Y, Komoto A, Kanazawa K. Fucoxanthin induces cell cycle arrest at G0/G1 phase in human colon carcinoma cells through up-regulation of p21WAF1/ Cip1. Biochim Biophys Acta, 2005; 1726(3):328-35.

Dragutinovic VV, Radonjic NV, Petronijevic ND, Tatic SB, Dimitrijevic IB, Radovanovic NS, et al. Matrix metalloproteinase-2 (MMP2) and -9 (MMP-9) in preoperative serum as independent prognostic markers in patients with colorectal cancer. Mol Cell Biochem, 2011; 355(12):173-8.

Hammond WA, Swaika A, Mody K. Pharmacologic resistance in colorectal cancer: a review. Ther Adv Med Oncol, 2016; 8(1):57-84.

Hosokawa M, Kudo M, Maeda H, Kohno H, Tanaka T, Miyashita

K. Fucoxanthin induces apoptosis and enhances the antiproliferative effect of the PPARgamma ligand, troglitazone, on colon cancer cells. Biochim Biophys Acta, 2004; 1675(1-3):113-9.

Huang H. Matrix metalloproteinase-9 (MMP-9) as a cancer biomarker and MMP-9 biosensors: recent advances. Sensors (Basel), 2018; 18(10).

Jonsson A, Hjalmarsson C, Falk P, Ivarsson ML. Stability of matrix metalloproteinase- 9 as biological marker in colorectal cancer. Med Oncol, 2018; 35(4):50.

Kim KN, Heo SJ, Kang SM, Ahn G, Jeon YJ. Fucoxanthin induces apoptosis in human leukemia HL-60 cells through a ROS-mediated Bcl-xL pathway. ToxicolIn Vitro, 2010; 24(6):1648-54.

Latchman J, Guastella A, Tofthagen C. 5-Fluorouracil toxicity and dihydropyrimidine dehydrogenase enzyme: implications for practice. Clin J Oncol Nurs, 2014; 18(5):581-5.

Lindequist U. Marine-derived pharmaceuticals - challenges and opportunities. Biomol Ther (Seoul), 2016;24(6):561-71.

Mackay AR, Ballin M, Pelina MD, Farina AR, Nason AM, Hartzler JL, Thorgeirsson UP. Effect of phorbol ester and cytokines on matrix metalloproteinase and tissue inhibitor of metalloproteinase expression in tumor and normal cell lines. Invasion Metastasis, 1992;12(3-4):168-84.

Maeda H, Kanno S, Kodate M, Hosokawa M, Miyashita K. Fucoxanthinol, metabolite of fucoxanthin, improves obesity-induced inflammation in adipocyte cells. Mar Drugs, 2015; 13(8):4799-813.

Martin LJ. Fucoxanthin and its metabolite fucoxanthinol in cancer prevention and treatment. Mar Drugs, 2015; 13(8):4784-98.

Moon JH, Kim JG, Sohn SK, Baek JH, Cho YY, Chae YS, Ahn BM, Kim SN, Lee SJ, Lee IT, Choi GS, Jun SH. Outpatient-basis chemotherapy of oxaliplatin, 5-fluorouracil, and leucovorin as first-line treatment for patients with metastatic or recurrent colorectal cancer. J Korean Med Sci, 2007; 22(3):400-4.
Mukai M, Okada K, Fukumitsu H, Yazawa N, Hoshikawa T, Tajima T, Hirakawa H, Ogoshi K, Makuuchi H. Efficacy of 5-FU/LV plus CPT-11 as first-line adjuvant chemotherapy for stage IIIa colorectal cancer. Oncol Rep, 2009; 22(3):621-9.

Murad H, Hawat M, Ekhtiar A, AlJapawe A, Abbas A, Darwish $\mathrm{H}$, Sbenati O, Ghannam A. Induction of G1-phase cell cycle arrest and apoptosis pathway in MDA-MB-231 human breast cancer cells by sulfated polysaccharide extracted from Laurenciapapillosa. Cancer Cell Int, 2016; $16: 39$.

National Comprehensive Cancer Network Guidelines for patients, colon cancer 2018. [ONLINE] Available via https://www.nccn. org/patients/guidelines/colon/files/assets/common/downloads/files/colon. pdf (Accessed 20 July 2019).

Parker WB, Cheng YC. Metabolism and mechanism of action of 5-fluorouracil. Pharmacol Ther, 1990; 48(3):381-95.

Rengarajan T, Rajendran P, Nandakumar N, Balasubramanian MP, Nishigaki I. Cancer preventive efficacy of marine carotenoid fucoxanthin: cell cycle arrest and apoptosis. Nutrients, 2013; 5(12):4978-89.

Shin YM, Han HS, Lim SW, Kim BC, Cheoi KS, Eum YO, Kim ST, Lee KH. Combination chemotherapy of oxaliplatin, 5-fluorouracil and low dose leucovorin in patients with advanced colorectal cancer. Cancer Res Treat, 2005; 37(5):284-9.

Sy AU, Lim E, Ka'opua LS, Kataoka-Yahiro M, Kinoshita Y, Stewart SL. Colorectal cancer screening prevalence and predictors among Asian American subgroups using Medical Expenditure Panel Survey National Data. Cancer, 2018; 124(Suppl 7):1543-51.

Van den Steen PE, Dubois B, Nelissen I, Rudd PM, Dwek RA, Opdenakker G. Biochemistry and molecular biology of gelatinase B or matrix metalloproteinase-9 (MMP-9). Crit Rev Biochem Mol Biol, 2002 37(6):375-536.

Van der Jeught K, Xu HC, Li YJ, Lu XB, Ji G. Drug resistance and new therapies in colorectal cancer. World J Gastroenterol, 2018; 24(34):3834-48.

Ye G, Lu Q, Zhao W, Du D, Jin L, Liu Y. Fucoxanthin induces apoptosis in human cervical cancer cell line HeLa via PI3K/Akt pathway, Tumour Biol. 2014; 35(11):11261-7.

Zhang C, Ma Q, Shi Y, Li X, Wang M, Wang J, Ge J, Chen Z, Wang Z, Jiang H. A novel 5-fluorouracil-resistant human esophageal squamous cell carcinoma cell line Eca-109/5-FU with significant drug resistance-related characteristics. Oncol Rep, 2017; 37(5):2942-54.

Zhang H, Tang Y, Zhang Y, Zhang S, Qu J, Wang X, Kong R, Han C, Liu Z. Fucoxanthin: a promising medicinal and nutritional ingredient. Evid Based Complement Alternat Med, 2015; 2015:723515.

How to cite this article:

Manmuan S, Manmuan P. Fucoxanthin enhances 5-FU chemotherapeutic efficacy in colorectal cancer cells by affecting MMP-9 invasive proteins. J Appl Pharm Sci, 2019; 9(12):007-014. 